\title{
BMJ open Are race-specific ERCC1 haplotypes in melanoma cases versus controls related to the predictive and prognostic value of ERCC1 N118N?
}

\author{
Rui Gao, ${ }^{1}$ Kelie M Reece, ${ }^{1}$ Tristan Sissung, ${ }^{2}$ Samuel H Fu, ${ }^{2}$ David J Venzon, ${ }^{3}$ \\ Eddie Reed, ${ }^{4}$ Shawn D Spencer, ${ }^{5}$ Douglas K Price, ${ }^{1,5}$ William D Figg ${ }^{1,2}$
}

To cite: Gao R, Reece KM, Sissung $\mathrm{T}$, et al. Are racespecific ERCC1 haplotypes in melanoma cases versus controls related to the predictive and prognostic value of ERCC1 N118N? BMJ Open 2013;3:e002030. doi:10.1136/bmjopen-2012002030

- Prepublication history and additional material for this paper are available online. To view these files please visit the journal online (http://dx.doi.org/10.1136/ bmjopen-2012-002030).

Received 29 August 2012 Revised 5 November 2012 Accepted 19 November 2012

This final article is available for use under the terms of the Creative Commons Attribution Non-Commercial 2.0 Licence; see http://bmjopen.bmj.com

For numbered affiliations see end of article

Correspondence to Dr William D Figg; wdfigg@helix.nih.gov

\section{ABSTRACT}

Objectives: Although it does not alter the ERCC1 phenotype, the ERCC1 500C>T (rs11615) polymorphism has undergone a myriad of investigations into its role as a marker for nucleotide excision repair (NER) function in different races, diseases and treatment outcomes. The goal of our study was to test the hypothesis that $500 \mathrm{C}>T$ is in linkage disequilibrium (LD) with causative alleles, and that these haplotypes are more frequent in Caucasians with melanoma than in healthy Caucasians or African Americans.

Design: In this case-control study, we selected racespecific ERCC1 single-nucleotide polymorphism (SNPs), conducted LD analysis with ERCC1 500C>T and compared the frequency of $E R C C 1$ diplotypes in Caucasians with melanoma $(n=165)$, healthy Caucasians ( $n=150)$ and healthy African Americans $(n=159)$. The haplotype was further studied using a fusion gene containing multiple ERCC1 SNPS.

Setting: Large cancer institute in the USA.

Participants: A total of 165 Caucasian melanoma patients, 159 healthy Caucasian controls and 159 African American healthy controls. Men and women were enrolled in the clinical trial; however, since the screening trial included prostate cancer screening in addition to screening for other cancers, only male controls were available.

Outcome measures: The outcome measures were melanoma risk in Caucasians, and LD between ERCC1 SNP, N118N and other race-specific allelic variants.

Results: When compared to ERCC1 500C $>T$ alone, a race-specific three-SNP variant haplotype in ERCC1 (comprised of rs11615, rs3212950 and rs3212948) was even more frequent in Caucasians with melanoma than in healthy Caucasians $(p=0.0034)$ or African Americans $(p<0.0001)$. A plasmid containing the variant haplotype was not differentially expressed. Conclusions: We demonstrate that ERCC1 500C>T participates in a previously characterised cancer-risk haplotype found more frequently in Caucasians, while LD is weak in African Americans; this haplotype appears to also be related to melanoma. It is therefore likely that $E R C C 1500 C>T$ is only a valid NER, disease or treatment outcome marker in Caucasians.

\section{ARTICLE SUMMARY}

Article focus

- Genetic variation in ERCC1 is thought to contribute to interindividual differences in the heritable risk and clinical progression of multiple forms of cancer.

- In this study, we used melanoma as a model disease to test the hypothesis that the ERCC1 $500 C>T$ polymorphism is more frequent in Caucasians with melanoma than in healthy Caucasians or African Americans.

\section{Key messages}

- ERCC1 500C $>T$ participates in a previously uncharacterised cancer-risk haplotype found in Caucasians and Asians; however, linkage disequilibrium between these alleles is significantly weaker in African Americans.

- In spite of the compendium of studies on ERCC1 N118N in multiple races, the N118N SNP is less likely to be important in African Americans. Thus, it is likely that ERCC1 500C>T can only be used as a predictive and prognostic marker for melanoma in Caucasians.

Strengths and limitations of this study

- Here, we study a novel race-specific haplotype for the most studied ERCC1 variant, N118N and characterise it in Caucasians, Asians and African Americans. This race-specific haplotype is better associated with melanoma incidence than ERCC1 $\mathrm{N} 118 \mathrm{~N}$ alone, suggesting that $\mathrm{N} 118 \mathrm{~N}$ is merely a passenger polymorphism while other allelic variants are causative.

- The limitation of this study was that little demographic data were available for the cases and controls; however, historical controls were compared to ensure that the present results were similar to previous studies.

\section{INTRODUCTION}

In humans, UV-induced and platinum-induced DNA lesions are removed from the genome via nucleotide excision repair (NER) mechanisms 
that are mediated, in part, by ERCC1. ${ }^{1}$ The gene encoding ERCC1 is highly polymorphic and confers significant variation in DNA-repair phenotypes. Consequently, genetic variation in ERCC1 is thought to contribute to interindividual differences in the heritable risk and clinical progression of multiple forms of cancer and/or outcome following DNA damaging therapy. ${ }^{2-9}$ A multitude of studies have focused on a particular ERCC1 polymorphism, 500C $>\mathrm{T}$ $(\mathrm{N} 118 \mathrm{~N}, \mathrm{rs} 11615)$, although meta-analyses of these data have concluded that there is either no consistency between studies, or that the N118N single-nucleotide polymorphism (SNP) does not generally have predictive power. ${ }^{10-13}$

Even the molecular mechanism behind the polymorphisms' contribution to disease risk and treatment outcome is unclear. Some studies suggest that the polymorphism confers a reduction in ERCC1 expression with less DNA repair (ie, a 'reduced NER' phenotype) ${ }^{6} 14$ while others have not observed a difference in ERCC1 expression or function. ${ }^{15}{ }^{16}$ Furthermore, inter-racial genetic variation in the NER pathway has been poorly studied in spite of known ethnic disparities arising from other germline genetic factors in multiple cancer types $^{17-24}$ and race-specific haplotypes in 19q13.3 (containing ERCC1, ERCC2, PPP1R13L and CD3EAP). Thus, broader haplotypes, rather than a single SNP in ERCC1, may confer broader race-specific differences in NER phenotypes. ${ }^{25}$

We previously showed that the putative reduced-NER phenotype conferred by the N118N SNP in ERCC1 is more prevalent in the European American (ie, Caucasian) population, compared to African Americans. ${ }^{26}$ We also showed that ERCC1 500C $>$ T alone does not itself confer any detectible expression or other NER phenotypic alterations ${ }^{15}$ and is only associated with satraplatin treatment outcome in Caucasians, not African Americans (personal communication, Figg et al, 2010). Results from the latter studies indicate that there are significant race-specific differences in linkage disequilibrium (LD) between this SNP and other nearby alleles that may be causative. Schierup et $a l^{25}$ showed that a commonly inherited haplotype in $19 \mathrm{q} 13.3$ is related to breast cancer incidence in Caucasians but not in individuals of African descent; however, they did not study ERCC1 rs11615 in spite of significant LD between rs11615 and the proposed risk haplotype. Therefore, we hypothesised that differences in a commonly inherited ERCC1 haplotype, in which rs11615 is included, underlie: (1) the inherited risk of developing certain tumours, (2) the response to platinum therapy and (3) the significant racial disparity in risk and treatment between Caucasians and African Americans in some diseases.

Consistent with this hypothesis, genetic variation in ERCC1 appears to be an important factor in risk, treatment and racial disparities in melanoma. It was previously shown that ERCC1 $500 C>T$ was linked to the risk of developing melanoma in Caucasians ${ }^{5}$ even though the $500 C>T$ SNP does not appear to alter ERCC1 phenotype. $^{15}$ Additionally, the race-specific haplotype organisation of
ERCC1 could be, in part, behind the following observations: (1) Caucasians have an approximate 20-fold increase in the incidence of melanoma compared to African Americans (http://www.cdc.gov/cancer/npcr/uscs/pdf/ 2002_USCS.pdf; last accessed 24 Aug 11) and (2) that African Americans who develop malignant melanoma have more aggressive disease and a significantly worse prognosis. ${ }^{27}$ Although differences in melanin expression are largely behind the former observation, it is possible that Caucasians harbour a genetic predisposition to UV-induced genotoxicity, and therefore malignant melanoma, whereas African Americans are less susceptible. However, African Americans may have a poorer prognosis following the development of malignant melanoma as a direct result of having increased DNA-repair capacity when platinum agents are administered; however, this latter issue has not been sufficiently investigated.

Using melanoma as a model disease, we studied the inheritance of ERCC1 diplotypes in cases and controls from the Caucasian population, and ascertained to what extent common haplotypes observed in Caucasians were also found in the healthy African American population.

\section{MATERIALS AND METHODS \\ Subjects}

All patients were European Americans with malignant melanoma, who were enrolled in a clinical trial being conducted at the National Cancer Institute. Samples were blinded; thus, no demographic information was available. Whole blood samples from healthy male volunteers who were screened for various cancers, including melanoma, (Valley Biomedical Inc, Winchester, Virginia, USA) were also analysed. For ethical considerations, only age and race data were available from these individuals. Informed consent was obtained from all subjects before trial participation, and the Institutional Review Board (IRB) also approved of the clinical trial, and all genotyping studies contained herein.

\section{Genotyping}

Plasma and/or whole blood were used to isolate genomic DNA according to the manufacturer's instructions using the Blood Kit (Qiagen, Valencia, California, USA). PCR was performed as described in our previous publication. ${ }^{15}$ The additional primers and PCR conditions were as follows: for rs3212948 and rs3212950, the primers are 5'-AGG AGA GAC GCC CAA CCA GG-3' and $5^{\prime}$-TGG CAC CAG GCC TTT CCT AAA G-3'. The annealing temperature was $66^{\circ} \mathrm{C}$. For rs3212929, the primers were 5'-TCA GAG AGC TGC AAG TTA GAA CAG TG-3' ${ }^{\prime}$ and $5^{\prime}$-TCA GAG AGG TGC AAG TTA GAA CAG TG-3 $3^{\prime}$ and the annealing temperature was $64^{\circ} \mathrm{C}$. Direct nucleotide sequencing PCR was conducted using the Big Dye Terminator Cycle Sequencing Ready Reaction kit V3.1 (Applied Biosystems, Foster City, California, USA) and an ABI Prism 3130 Genetic 
Analyzer according to the manufacturer's instructions. Complete genotyping data were not available for all patients.

\section{Statistical considerations}

Fisher's exact test was used to evaluate the differences between healthy volunteers and patients with malignant melanoma in the frequency distributions of genotype of the selected polymorphisms. The Hardy-Weinberg equilibrium was tested using the $\chi^{2}$ test. If we assume that each allele or haplotype increases the outcome risk by the same OR, then with allele frequencies in the range of $0.40-0.50$ in controls, and with the highest observed numbers of alleles in table 1 , an $\mathrm{OR}>1.71$ would be found significant with $>80 \%$ power in a two-tailed Fisher's exact test at the $\alpha=0.05$ level. Since the power analysis was done post hoc, and therefore used experimentally derived parameter values, this information is only intended to demonstrate that statistical power (eg, the probability of rejecting the null hypothesis when it is not true) was within generally accepted limits. Moreover, since we analysed linked alleles, a strict Bonferroni correction would be too stringent; we therefore set nominal significance below $\mathrm{p}=0.01$ to reduce the possibility of assigning significance to a false-positive result.

Haploview (http://www.broadinstitute.org/scientificcommunity/science/programs/medical-and-populationgenetics/haploview/haploview, last accessed $23 \mathrm{Feb} 12^{28}$ ) was used to calculate allele frequencies. Phase V.2.1.1 ${ }^{29} 30$ was used to calculate the most likely haplotypes and diplotypes for the investigated ERCC1 polymorphisms in melanoma patients and healthy subjects. Haploview software was also used to perform LD assessments and to define the haplotype blocks by the incorporated CI method as described by Gabriel. ${ }^{31}$ The differences in haplotype allele and diplotype frequency distributions were evaluated by Fisher's exact test.

\section{Cell culture}

The SK-Mel-5 cell line was obtained from American Type Culture Collection (Manassas, Virginia, USA). Cells were maintained in a humidified incubator at $37^{\circ} \mathrm{C}$ equilibrated with $5 \% \mathrm{CO}_{2}$ and $95 \%$ air in an RPMI-1640 medium containing $10 \%$ fetal bovine serum and $1 \%$ penicillin-streptomycin.

\section{Plasmid constructs}

The gene fragments containing ERCC1 intron 3 and exon 4 regions were amplified from patients with melanoma who had either a homozygous wild-type or homozygous variant haplotype, as defined in the association analysis. The PCR products were cloned in-frame between the EcoRI and KpnI sites of the pCMV-HA and pCMV-Myc vector set. The vector pair was co-transfected into SK-Mel-5 cells using Lipofectamine 2000 (Invitrogen, Carlsbad, California, USA) when cells were $70 \%$ confluent as per the manufacturer's instructions.

\section{Quantitative reverse transcription-PCR}

The expression of HA or Myc-tagged ERCC1 fragments was measured by quantitative reverse transcription-PCR using the Stratagene Mx3005P Real-Time PCR System (La Jolla, California, USA). Briefly, cells were lysed and the total RNA was collected $48 \mathrm{~h}$ after transfection using the RNeasy Mini Kit (Qiagen). The total RNA extracted was reverse-transcribed using $\mathrm{RT}^{2}$ First Strand Kits (SABiosciences, Frederick, Maryland, USA). Each sample was analysed in duplicate and the results are an average of four analyses. Analysis of mRNA expression was conducted using primers specific to the tag and the ERCCI sequence, and normalised to a human reference gene GAPDH by RT ${ }^{2}$ qPCR Primer Assay (SABiosciences).

\section{RESULTS}

Frequency of genetic variants in DNA excision repair genes in melanoma cases and healthy controls

We first genotyped polymorphisms in genes involved in the NER (ie, ERCC1 (N118N and C8092A) and XPD (K751Q)) or BER (ie, XRCC1 (R399Q and R194W) and PARP1 (V762A)) pathways in patients with melanoma $(n=165)$ and compared the allele frequency of these SNPs to a cohort of healthy individuals $(n=150$ Caucasians and $\mathrm{n}=159$ African Americans; methods included in ref. $^{26}$ ). All polymorphisms were in Hardy-Weinberg equilibrium in both the melanoma

\begin{tabular}{|c|c|c|c|c|c|}
\hline $\begin{array}{l}\text { SNP } \\
\text { name }\end{array}$ & Allele & $\begin{array}{l}\text { Caucasians with melanoma } \\
\text { (Counts (allele frequency)) }^{*}\end{array}$ & $\begin{array}{l}\text { Caucasian controls (Counts } \\
\text { (allele frequency)) }\end{array}$ & OR (95\% Cl) & $\begin{array}{l}p \\
\text { Value }\end{array}$ \\
\hline \multirow[t]{2}{*}{ rs11615 } & C & $104(0.33)$ & $99(0.46)$ & 1.69 (1.19 to 2.42$)$ & 0.0047 \\
\hline & $\mathrm{T}$ & $208(0.67)$ & $117(0.54)$ & & \\
\hline \multirow[t]{2}{*}{ rs3212950 } & C & $87(0.30)$ & $83(0.44)$ & 1.79 (1.22 to 2.62$)$ & 0.0033 \\
\hline & G & $201(0.70)$ & $107(0.56)$ & & \\
\hline \multirow[t]{2}{*}{ rs3212948 } & C & $86(0.30)$ & $86(0.45)$ & $1.94(1.33$ to 2.84$)$ & 0.0007 \\
\hline & G & $202(0.70)$ & $104(0.55)$ & & \\
\hline \multirow[t]{2}{*}{ rs3212929 } & G & $287(0.99)$ & $183(0.96)$ & 10.98 (1.34 to 90.0$)$ & 0.0077 \\
\hline & $\mathrm{T}$ & $1(0.003)$ & $7(0.037)$ & & \\
\hline
\end{tabular}


cases and controls $(\mathrm{p}>0.05)$; thus, the potential for tumour DNA to be genotyped in plasma samples was unlikely. Our control allele frequency was not significantly different $(p>0.05)$ from a cohort of 1358 USA Caucasians screened by Zhou et al..$^{13}$ Of the aforementioned SNPs, only ERCC1 500C $>$ T (in exon 4) was found to be more frequent in patients diagnosed with melanoma (OR $(95 \% \mathrm{CI})=1.69 \quad(1.19$ to $2.42 ; \mathrm{p}=0.0047))$. While the statistically significant OR implies that the variant allele confers risk of developing melanoma, given that demographics data were not available, other factors may underlie this association. Nonetheless, it is notable that these data are very similar to a previous study that reported an OR of 1.59 for this same polymorphism in Caucasians. ${ }^{5}$

Since we were previously unable to detect any differences in ERCC1 phenotype based on the 500C>T SNP alone, ${ }^{15}$ we reasoned that the $500 C>T$ SNP was in LD with causative alleles and that the frequency of these haplotypes would be even higher in Caucasian melanoma patients versus controls. Furthermore, our previous studies indicated that Caucasians and African Americans inherit different haplotypes ${ }^{26}$ and that the ERCC1 $500 C>T S N P$ is a poor marker of response to platinum in African Americans but is strongly related to survival following platinum agents in Caucasians (unpublished data). We therefore selected candidate SNPs for risk and LD analysis that had distinct frequency differences in Caucasians and African Americans according to the NCBI SNP database. As indicated in online supplementary table S1, the race-specific SNPs included rs3212950 $(\mathrm{C}>\mathrm{G})$ and rs3212948 $(\mathrm{G}>\mathrm{C})$ in intron 3, and rs3212929 $(\mathrm{T}>\mathrm{G})$ in the $5^{\prime}$ region of the gene. Only the rs3212950 and 3212948 SNPs were determined to be in strong LDwith ERCC1 500C>T in the present Caucasian population. Consistent with our hypothesis, all of these SNPs were more frequent in the melanoma cohort versus healthy controls (OR (95\% CI) $>1.69$ (1.19 to 2.42); $\mathrm{p}<0.0077$; see table 1$)$.

\section{Frequency of ERCC1 diplotypes in Caucasians with melanoma, healthy Caucasians and African American controls}

We used Haploview to determine the most likely haplotypes in cases and controls. There were three haplotypes in the Caucasian population that comprised three polymorphisms (ie, rs11615, rs3212950 and rs3212948): CCG, TGC and CGC. These haplotypes were inherited in five different diplotype combinations (see table 2). The majority of individuals carried TGC and/or CCG alleles with the exception of seven patients and two healthy volunteers who carried rare diplotypes composed of CGC alleles; these were excluded from further analysis. The 'heterozygous' diplotype, CCG-TGC, was not significantly more frequent in melanoma; however, the TGCTGC diplotype was significantly more frequent in melanoma than in controls $(\mathrm{OR}=2.88, \mathrm{p}=0.0119$, table 2). Again, the OR must be interpreted with caution, but this
Table 2 Race-specific ERCC1 diplotypes frequency in patients with melanoma versus controls

\begin{tabular}{lllll}
\hline \multicolumn{5}{c}{$\begin{array}{l}\text { Caucasian melanoma cases vs Caucasian } \\
\text { controls* }\end{array}$} \\
\cline { 2 - 5 } Cases & $\begin{array}{l}\text { Controls } \\
\text { Diplotype }\end{array}$ & $\begin{array}{l}\text { (\%) } \\
\text { (\%) }\end{array}$ & OR (95\% Cl) & p Value \\
\hline CCG-CCG & $16(11)$ & $18(20)$ & N/A & N/A \\
CCG-TGC & $50(35)$ & $45(49)$ & $1.25(0.57$ to 2.74) & 0.69 \\
TGC-TGC & $69(49)$ & $27(29)$ & $2.88(1.28$ to 6.45$)$ & 0.012 \\
TGC-CGC & $5(4)$ & $1(1)$ & Not tested & N/A \\
CCG-CGC & $2(1)$ & $1(1)$ & Not tested & N/A \\
\hline${ }^{*}$ Complete genotyping data were not available in all patients.
\end{tabular}

observation suggests that this diplotype may be associated with greater melanoma risk than each of the individual genotypes considered alone.

\section{ERCC1 diplotypes in Caucasians versus African Americans}

The healthy Caucasian population carries a greater proportion of ERCC1 variant alleles that may be linked to melanoma than does the African American population ( $p<0.0001$; online supplementary table S1). Moreover, the African American population has significantly less LD between $500 C>T$ and the other two SNPs in the 'CCG/TGC' diplotype than does the Caucasian population $\left(r^{2}=0.52\right.$ for $r s 11615$ linked to $r s 3212950$ and rs3212948 in African Americans vs $r^{2}>0.86$ in Caucasians, respectively; table 3). Therefore, the ERCC1 $500 \mathrm{C}>\mathrm{T}$ SNP (ie, rs11615) does not appear to be a strong marker for risk diplotypes (ie, a 'tagging SNP') in the African American population while it is a strong tagging SNP for risk diplotypes in the Caucasian population. Since African Americans have low LD between these alleles (see table 3), we were unable to ascertain the inheritance of haplotypes in this population for diplotype comparison.

\section{Effect of ERCC1 haplotypes on transcription}

In order to determine if the intronic and exonic regions (all SNPs in intron 3 and exon 4) containing the haplotype (variant) are responsible for the altered transcription of ERCC1, the homozygous wild-type and variant haplotypes, CCG-CCG and TGC-TGC of ERCC1 intron 3 and exon 4, respectively, were cloned in-frame between the EcoRI and KpnI sites of the pCMV-HA and pCMV-Myc vectors, followed by transfection into a skin melanoma cell line, SK-Mel-5. The level of transcripts of the wild-type fusion gene was 1.16 -fold higher than the variant genotype, although this was not significant ( $p=0.62$, figure 1$)$. Thus, it appears that the present SNPs are not themselves functional; but rather, longrange regulation linked to the present haplotype is likely responsible for changes in ERCC1 transcription. 
Table 3 Pairwise linkage measurements in the Caucasian vs African American populations

\begin{tabular}{lllll}
\hline SNP1 & SNP2 & $\mathbf{r}^{\mathbf{2}}$ & & \\
\cline { 3 - 5 } & & Patients with melanoma & Caucasian controls & African American controls \\
\hline rs11615 & rs3212950 & 0.86 & 0.92 & 0.52 \\
rs11615 & rs3212948 & 0.88 & 0.90 & 0.52 \\
rs11615 & rs3212929 & 0.01 & 0.02 & 0.10 \\
rs3212950 & rs3212948 & 0.98 & 0.94 & 1 \\
rs3212950 & rs3212929 & 0.01 & 0.02 & 0.08 \\
rs3212948 & rs3212929 & 0.01 & 0.02 & 0.08 \\
\hline
\end{tabular}

\section{LD block analysis in the HapMap Project}

While transcriptional analysis indicated that the CCG/ TGC diplotype is not predictive of expression, there is a larger haplotype block upstream of rs11615 in Caucasians that contains other potential causative alleles. This LD block consists of nine upstream alleles in Caucasians (CEU+TSI) participating in the HapMap Project (rs3212950 and rs3212948 are not ascertained by HapMap ${ }^{32}$ ). Specifically, there are three additional SNPs in the ERCC1 gene that are in strong $\mathrm{LD}$ with $500 C>T$ (ie, rs3212961, rs3212980 and rs3212986). Within the same LD block, there are seven more SNPs within or adjacent to the CD3EAP (shares sequence with ERCC1) or PPP1R13L genes (ie, rs2336219, rs762562, rs735482, rs1046282, rs10412761, rs4803817 and rs4803816; figure 2A). Notably, rs735482, rs3212986 and rs3212961 were implicated in a Caucasian-specific breast cancer risk haplotype by Schierup et $a l^{25}$ There were no alleles in strong LD with ERCC1 500C>T in the African (ASW) population (figure 2B). Interestingly, although a specific

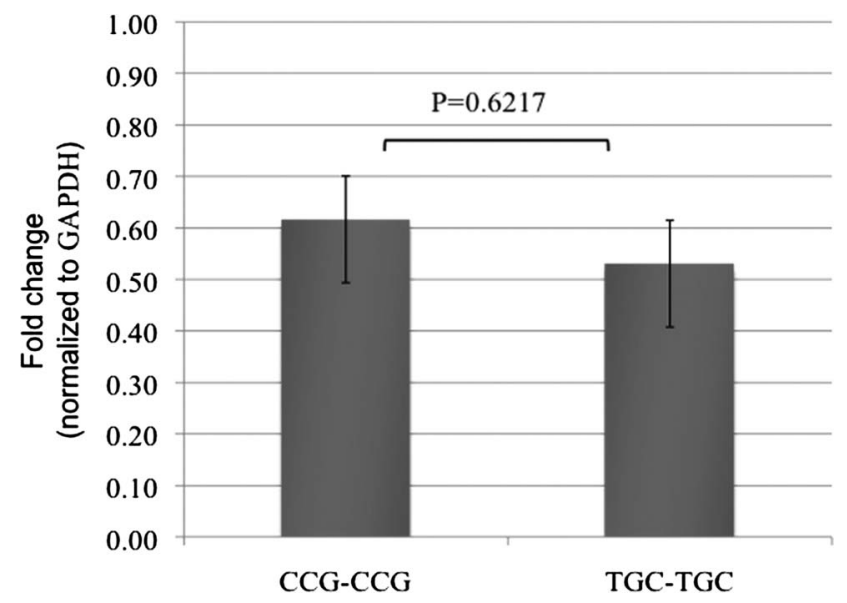

Figure 1 Relative abundance of transcripts of the two fusion genes with wild-type or variant genotypes. The fusion genes with different ERCC1 genotypes were cloned in frame between the EcoRI and Kpnl sites of the pCMV-HA and pCMV-Myc vector set. The relative abundance of the transcripts was normalised to the housekeeping gene GAPDH. The numbers on the bars indicate fold change of transcripts relative to GAPDH. The level of transcripts of the wild-type fusion gene is 1.16-fold higher than the variant genotype $(p=0.62)$.
LD block was not defined with ERCC1 500C>T, strong LD was also observed in the Asian $(\mathrm{CHB}+\mathrm{JPT})$ population (figure 2C) with the same alleles that were observed in the Caucasian population.

\section{DISCUSSION}

Using melanoma as a model disease, the present study indicates that while a multitude of studies have focused on the ERCC1 $500 C>T(\mathrm{~N} 118 \mathrm{~N}$ and rs11615) SNP in risk/ treatment associations in different races, ${ }^{2-9}$ the $500 C>T$ is merely a tagging SNP denoting a larger haplotype structure in the Caucasian population that appears to be related to heritable susceptibility to certain forms of cancer. ${ }^{25}$ With respect to the African American population, it is likely that associations with ERCC1 50OC>T and risk/ treatment have arisen due to racial admixture, and that the $500 C>T S N P$ is a poor marker for haplotypes (or diplotypes) that may alter NER phenotype in this population.

Our results with respect to the ERCC1 500C>T Tallele are remarkably similar to a previously published melanoma risk assessment in Caucasians conducted by Povey $e t a l^{5}$; however, they did not consider multiple race-specific alleles in ERCC1 that are likely associated with the incidence of melanoma in Caucasians. Therefore, the data presented herein suggest that future investigations studying the risk and treatment of melanoma in Caucasians should consider race-specific ERCC1 haplotypes instead of simply focusing on the $500 C>T$ polymorphism in isolation. Moreover, the $500 \mathrm{C}>\mathrm{T} \mathrm{SNP}$ is likely to have less prognostic and predictive value in African Americans and Africans than it does in Caucasians and Asians. We also suggest that this observation can be extrapolated to other disease types. For instance, the 500C $>T$ polymorphism has been heavily studied in breast, colorectal, gastric, glioma, lung and prostate cancers as both a prognostic marker and a predictive marker for platinum therapeutic outcomes in a multitude of individuals with different racial backgrounds. $^{2-9} 25$

The present data also lead to the conclusion that investigators studying the molecular genetic consequences of ERCC1 polymorphisms should consider the LD between $500 C>$ Tand many upstream polymorphisms as these could have major effects on ERCC1 transcription and function. HapMap analysis in Caucasians (CEU+TSI) indicated that there are at least five additional upstream polymorphisms 
(a)

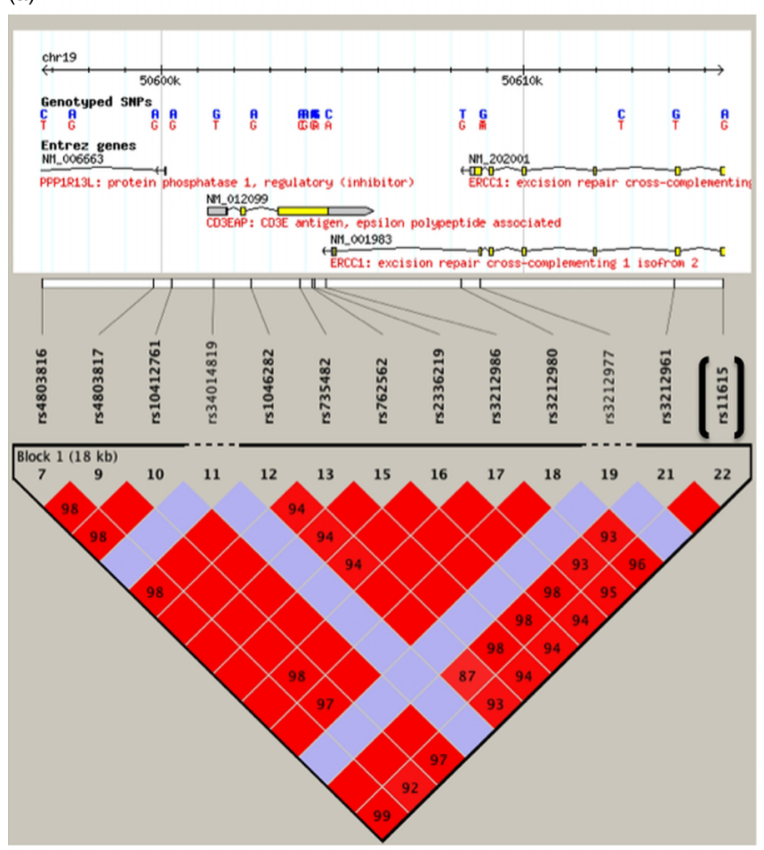

(b)

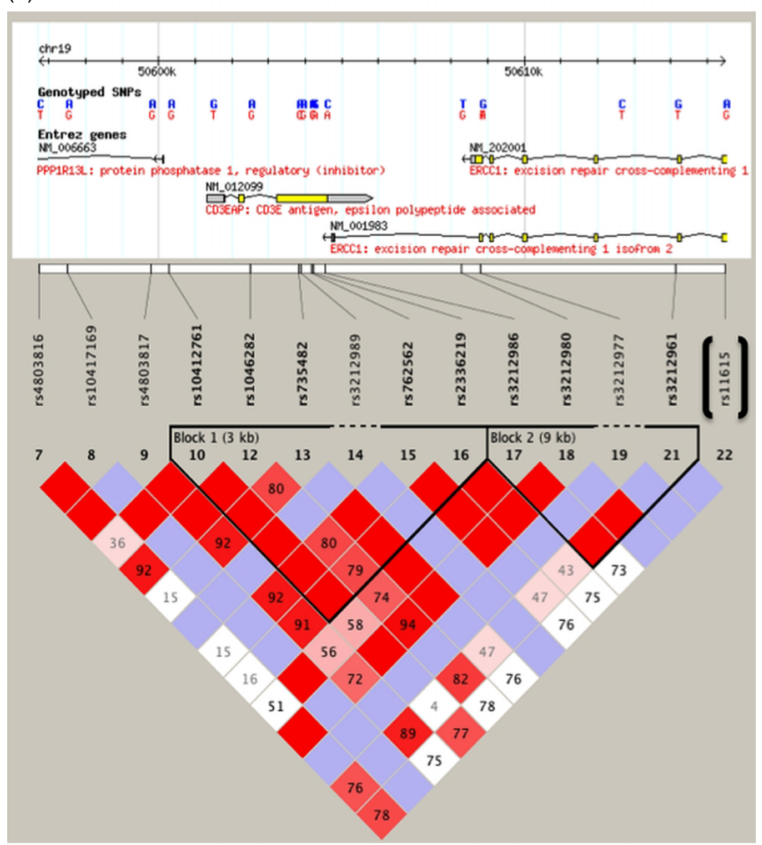

(c)

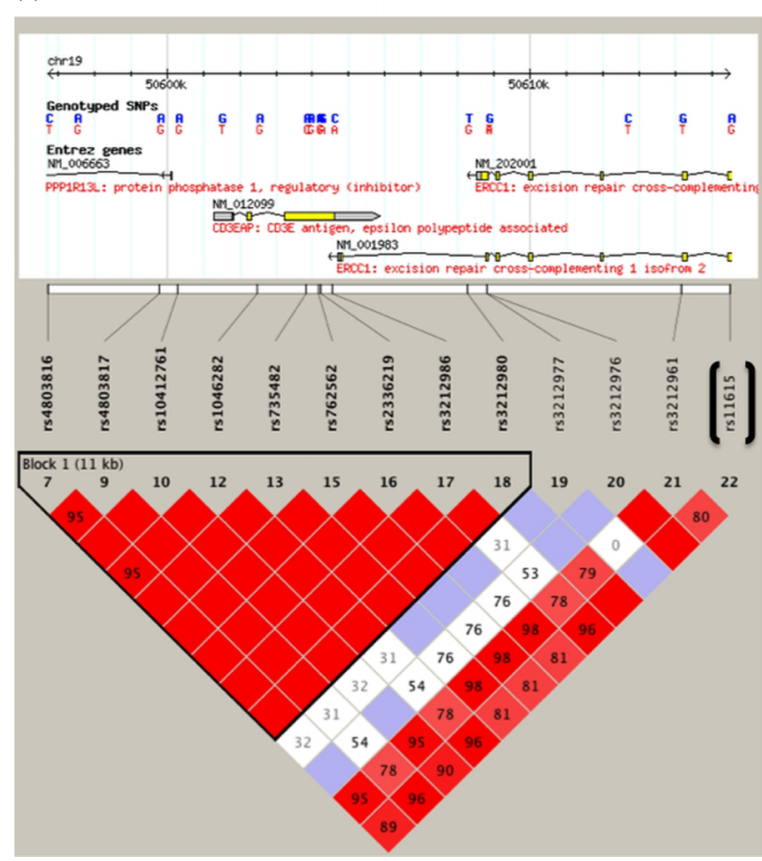

Figure 2 Linkage disequilibrium between rs11615 (500C>T)* and other ERCC1 alleles in the HapMap Project for $(\mathrm{A})$ Caucasians (CEU+TSI), (B) Africans (ASW) and (C) Asians (CHI+JPT). *Note that the HapMap output in Haploview lists bases on the opposite strand of ERCC1: therefore $500 C>T$ is equivalent to $E R C C 1500 G>A$.

within, or directly adjacent to, the ERCC1 gene that are linked to $500 C>T$. Furthermore, there are five SNPs in the same LD block that are within or adjacent to the CD3EAP or PPP1R13L genes. Interestingly, strong LD with adjacent alleles was also observed in the Asian $(\mathrm{CHB}+\mathrm{JPT})$ population, but there were no alleles in strong LD with ERCC1 $500 C>T$ in the African (ASW) population. It is therefore likely that the phenotype-altering consequences of $500 C>T$ directly result from other variants in ERCC1 or in other adjacent genes in Caucasians. ${ }^{25}$ Moreover, it is likely that the causative allele(s) may be found by studying alleles that are linked to $500 C>T$ in Caucasians and Asians, but not in Africans.

NER is involved in the aetiology, clinical progression and treatment of a great number of diseases; therefore, understanding the molecular basis for NER phenotype variation caused by genetic variants in ERCC1 is anticipated to lead to a number of advancements in medicine. This endpoint is unlikely if future studies continue to only focus on a single variant in ERCC1 (ie, 500C $>\mathrm{T}$ ) 
that does not appear to have phenotypic consequences in isolation. ${ }^{10-1315}$ ERCC1 phenotypic variation is likely to be different among races, and variants that more accurately predict outcomes in the African American population must be established. It is therefore hoped that the present study will lead to a more informed approach to the study of ERCC1 in world populations suffering from disease.

\section{Author affiliations}

${ }^{1}$ Molecular Pharmacology Section, Medical Oncology Branch, Center for Cancer Research, Bethesda, Maryland, USA

${ }^{2}$ Clinical Pharmacology Core, Medical Oncology Branch, Center for Cancer Research, Bethesda, Maryland, USA

${ }^{3}$ Biostatistics \& Data Management Section, National Cancer Institute, Bethesda, Maryland, USA

${ }^{4}$ Mitchell Cancer Institute, University of South Alabama, Mobile, Alabama, USA

${ }^{5}$ Clinical Pharmacology Program, SAIC-Frederick, NCI-Frederick, Frederick, Maryland, USA

Acknowledgements The authors would like to thank Dr Steven Rosenberg $(\mathrm{NCl})$ for providing us with the samples of patients with melanoma. This study was supported in part by the Intramural Research Program of the National Institutes of Health, National Cancer Institute, Bethesda, Maryland, USA.

Contributors RG and TS conceived the idea for the study and were responsible for the design of the study as well as the data analysis. RG did the bulk of the experimentation and produced the tables and graphs. RG, KMR and TS provided input into the data analyses. KMR worked with $R G$ on the experiment shown in figure 1. The initial draft of the manuscript was prepared by $R G$ and then circulated repeatedly among all authors for critical revision. RG was responsible for data acquisition and KMR, TS, SHF, DJV, ER, SDS, DKP and WDF contributed to the interpretation of the results. DV is a biostatistician and was the primary data analyst. All authors helped to interpret the data and critically revise successive drafts of the manuscript.

Funding This work was supported in part by the Intramural Research Program of the National Institutes of Health, National Cancer Institute, Bethesda, MD, USA.

Competing interests None.

Ethics approval Institutional Review Board of the NIH.

Provenance and peer review Not commissioned; externally peer reviewed.

Data sharing statement There are no additional data available.

\section{REFERENCES}

1. Reed E. Nucleotide excision repair and anti-cancer chemotherapy. Cytotechnology 1998;27:187-201.

2. Liu Y, Shete S, Hosking F, et al. Genetic advances in glioma: susceptibility genes and networks. Curr Opin Genet Dev 2010 20:239-44.

3. Kiyohara C, Yoshimasu K. Genetic polymorphisms in the nucleotide excision repair pathway and lung cancer risk: a meta-analysis. Int $J$ Med Sci 2007;4:59-71.

4. Mocellin S, Verdi D, Nitti D. DNA repair gene polymorphisms and risk of cutaneous melanoma: a systematic review and meta-analysis Carcinogenesis 2009;30:1735-43.

5. Povey JE, Darakhshan F, Robertson K, et al. DNA repair gene polymorphisms and genetic predisposition to cutaneous melanoma. Carcinogenesis 2007;28:1087-93.

6. Woelfelschneider A, Popanda O, Lilla C, et al. A distinct ERCC1 haplotype is associated with mRNA expression levels in prostate cancer patients. Carcinogenesis 2008;29:1758-64.

7. Liu D, O'Day SJ, Yang D, et al. Impact of gene polymorphisms on clinical outcome for stage IV melanoma patients treated with biochemotherapy: an exploratory study. Clin Cancer Res 2005;11:1237-46.
8. Wei SZ, Zhan P, Shi MQ et al. Predictive value of ERCC1 and XPD polymorphism in patients with advanced non-small cell lung cancer receiving platinum-based chemotherapy: a systematic review and meta-analysis. Med Oncol 2010;28:315-21.

9. Yin M, Yan J, Martinez-Balibrea E, et al. ERCC1 and ERCC2 polymorphisms predict clinical outcomes of oxaliplatin-based chemotherapies in gastric and colorectal cancer: a systemic review and meta-analysis. Clin Cancer Res 2011;17:1632-40.

10. Wei HB, Lu XS, Shang LH, et al. Polymorphisms of ERCC1 C118T/ C8092A and MDR1 C3435T predict outcome of platinum-based chemotherapies in advanced non-small cell lung cancer: a meta-analysis. Arch Med Res 2011;42:412-20.

11. Yu D, Shi J, Sun T, et al. Pharmacogenetic role of ERCC1 genetic variants in treatment response of platinum-based chemotherapy among advanced non-small cell lung cancer patients. Tumour Biol 2012;33:877-84

12. Zhang L, Wang J, Xu L, et al. Nucleotide excision repair gene ERCC1 polymorphisms contribute to cancer susceptibility: a meta-analysis. Mutagenesis 2011;27:67-76.

13. Zhou W, Liu G, Park S, et al. Gene-smoking interaction associations for the ERCC1 polymorphisms in the risk of lung cancer. Cancer Epidemiol Biomarkers Prev 2005;14:491-6.

14. Chang PM, Tzeng CH, Chen PM, et al. ERCC1 codon $118 \mathrm{C} \rightarrow \mathrm{T}$ polymorphism associated with ERCC1 expression and outcome of FOLFOX-4 treatment in Asian patients with metastatic colorectal carcinoma. Cancer Sci 2009;100:278-83.

15. Gao R, Reece K, Sissung T, et al. The ERCC1 N118N polymorphism does not change cellular ERCC1 protein expression or platinum sensitivity. Mutat Res 2011;708:21-7.

16. Huang ZH, Hua D, Du X, et al. ERCC1 polymorphism, expression and clinical outcome of oxaliplatin-based adjuvant chemotherapy in gastric cancer. World J Gastroenterol 2008;14:6401-7.

17. Zabaleta J, Schneider BG, Ryckman K, et al. Ethnic differences in cytokine gene polymorphisms: potential implications for cancer development. Cancer Immunol Immunother 2008;57:107-14.

18. Taioli E, Flores-Obando RE, Agalliu I, et al. Multi-institutional prostate cancer study of genetic susceptibility in populations of African descent. Carcinogenesis 2011;32:1361-5

19. Sharma S, Cao X, Wilkens LR, et al. Well-done meat consumption, NAT1 and NAT2 acetylator genotypes and prostate cancer risk: the multiethnic cohort study. Cancer Epidemiol Biomarkers Prev 2010;19:1866-70.

20. Morrison C, Miecznikowski J, Darcy KM, et al. A GOG 210 aCGH study of gain at 1q23 in endometrioid endometrial cancer in the context of racial disparity and outcome. Genes Chromosomes Cancer 2010;49:791-802.

21. Sarkissyan M, Mishra DK, Wu Y, et al. IGF gene polymorphisms and breast cancer in African-American and Hispanic women. Int $\mathrm{J}$ Oncol 2011;38:1663-73.

22. Levy DE, Byfield SD, Comstock CB, et al. Underutilization of BRCA1/2 testing to guide breast cancer treatment: black and Hispanic women particularly at risk. Genet Med 2011;13:349-55.

23. Berger FG. The interleukin-6 gene: a susceptibility factor that may contribute to racial and ethnic disparities in breast cancer mortality. Breast Cancer Res Treat 2004;88:281-5.

24. Polite BN, Dignam JJ, Olopade OI. Colorectal cancer and race: understanding the differences in outcomes between African Americans and whites. Med Clin North Am 2005;89:771-93.

25. Schierup $\mathrm{MH}$, Mailund $\mathrm{T}$, $\mathrm{Li} \mathrm{H}$, et al. Haplotype frequencies in a sub-region of chromosome 19q13.3, related to risk and prognosis of cancer, differ dramatically between ethnic groups. BMC Med Genet 2009;10:20.

26. Gao R, Price DK, Sissung T, et al. Ethnic disparities in Americans of European descent versus Americans of African descent related to polymorphic ERCC1, ERCC2, XRCC1, and PARP1. Mol Cancer Ther 2008;7:1246-50.

27. Kabigting FD, Nelson FP, Kauffman CL, et al. Malignant melanoma in African-Americans. Dermatol Online J 2009;15:3.

28. Barrett JC, Fry B, Maller J, et al. Haploview: analysis and visualization of LD and haplotype maps. Bioinformatics 2005;21:263-5.

29. Stephens M, Scheet P. Accounting for decay of linkage disequilibrium in haplotype inference and missing-data imputation. Am J Hum Genet 2005;76:449-62.

30. Stephens M, Donnelly P. A comparison of bayesian methods for haplotype reconstruction from population genotype data. Am J Hum Genet 2003;73:1162-9.

31. Gabriel S. Variation in the human genome and the inherited basis of common disease. Semin Oncol 2006;33(6 Suppl 11):S46-9.

32. International-Hapmap-Consortium. The International HapMap Project. Nature 2003;426:789-96. 